
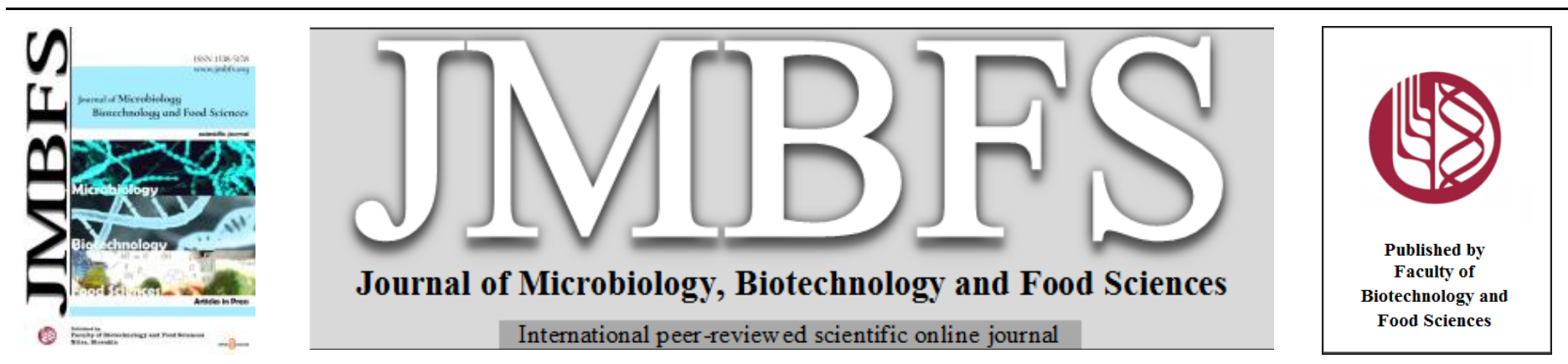

\title{
TRIAZOLE RESISTANCE IN ASPERGILLUS FUMIGATUS-A COMPREHENSIVE REVIEW
}

\author{
Khushbu Wadhwa, Hardeep Kaur*
}

Address(es):

Department of Zoology, Ramjas College, University of Delhi, Delhi, India.

*Corresponding author: hardeepkaur@ramjas.du.ac.in ; hkaur53d@gmail.com

https://doi.org/10.55251/jmbfs.4159

ARTICLE INFO

Received 3. 1. 2021

Revised 15. 8. 2021

Accepted 3. 9. 2021

Published 1. 2. 2022

Review

OPEN $\partial_{\text {ACCESS }}$

\begin{abstract}
Aspergillus fumigatus is a common ubiquitous fungal pathogen present in the environment including soil, decaying leaves, compost, grain crops and household dust. It can turn opportunistic in immunocompromised individuals causing allergic diseases, respiratory problems and invasive blood stream infections. Being saprophytic, it propagates by producing minute grey-green conidia that can become airborne and reach humans. In majority of cases, aspergillosis due to this organism can heal with treatment by antifungals. The azole compounds, one of the most important antifungal drugs, generally target the 14- $\alpha$ sterol demethylase enzyme, encoded by the cyp51A gene of the fungus, causing impaired membrane ergosterol synthesis. However in recent years the situation has turned grave due to development of azole resistant fungal species both in the environment as well as in the clinics. The main mechanism of resistance in A. fumigatus involves incorporation of tandem repeats in the promoter region of cyp51A, along with point mutations in the gene sequence. Resistant isolates tend to arise either due to excessive use of azole fungicides in the agricultural fields, from where they reach human through sporulation; or by prolonged treatment of patients with azole drugs. The early diagnosis of aspergillosis is very crucial for successful treatment of a patient. The article brings forth a comprehensive study of this organism, the mechanism of development and spread of azole resistant fungal isolates, genetic basis of development of azole resistance, detection of resistant strains and finally drugs used to combat the fungal infection.
\end{abstract}

Keywords: Antifungal, Aspergillosis, Azoles, CYP51A

\section{INTRODUCTION}

A. fumigatus is a saprophytic fungus that grows on dead and decaying parts of the organic matter. It is not a primary pathogen; however the use of immunosuppressive therapies or prolonged use of antifungals can turn it into an opportunistic fungal pathogen (Chowdhary et al., 2013). The genus Aspergillus is divided into five sections- fumigati, nidulante, flavi, nigri, and terrei, but only few species such as A. fumigatus and A. flavus are known to be the major pathogenic fungi in humans and animals. A. fumigatus is the most common airborne opportunistic fungal pathogen. Individuals suffering from asthma or cystic fibrosis are at a major risk to develop allergic bronchopulmonary aspergillosis. Invasive aspergillosis (IA) represents the severe form of the disease and targets mainly immunocompromised patients (Van de veerdonk $\boldsymbol{e t}$ al., 2017; Garcia rubio et al., 2017). Treatment options are limited to only three antifungal classes such as polyenes (amphotericin B), azole drugs and echinocandins. Triazole compound which are the most commonly used azoles, include itraconazole, voriconazole and posaconazole that represents the first line of therapy for the treatment of aspergillosis. However, azole resistant isolates exhibits a poor clinical outcome. Inhibition of CYP51A enzyme results in the significant loss of sterol content in the A. fumigatus cell (Dhingra and Cramer, 2017). It has been revealed that G54 and M220 are the two important hotspots for the development of azole resistant mutation in cyp51A (Mellado et al., 2004). Presence of tandem repeats in the promoter region of cyp51A along with point mutations in the azole binding site of the enzyme, causes reduced sensitivity of the fungi to azoles. The agricultural use of azole fungicides is thought to be a major route responsible for the emergence and spread of TR34/L98H and TR46/Y121F/T289A mutant isolates (Chowdhary et al., 2012a). Apart from alterations in cyp51A gene, non-cyp51A mutations causing drug resistance have also been reported in clinical isolates (Fraczek et al. 2013; Hagiwara et al., 2018; Gsaller et al., 2016; Camps et al., 2012a,b). The development of azole resistance among clinical isolates imparts a big challenge for the treatment of Aspergillus-related disease and demands a strong need for the development of alternative antifungal compounds.

\section{BIOLOGY OF A. FUMIGATUS}

A. fumigatus has enormous amount of ability to adapt to diverse environmental conditions. This property makes it a common pathogenic organism in human host. It produces millions of spores that ensure its survival and dispersal. The development of infection in human hosts starts through the inhalation of conidia (asexual spores) and its deposition in bronchioles and alveoli of the lungs. The conidia of A. fumigatus appear to be bluish-green or grey-green in color and have a globose to sub-globose like structure. These asexual spores are produced on a specialized hyphal structure called as conidiophores. They are hydrophobic in nature and their dispersal by air currents occurs very easily. The size of conidia plays an important role in the development of infection. The average size of Aspergillus conidia is about $2-3 \mu \mathrm{m}$ that is best suited for its deposition in the lungs, though large-sized conidia of A. flavus and A. niger is removed by the movement of mucociliary membrane in the lungs. In immunocompromised patients, the mucosal membrane of lung is not able to clear out inhaled conidia which can further lead to fungal growth and colonization (Dagenais and Keller, 2009). Apart from this, Aspergillus shows thermotolerance. It can grow well at $37^{\circ} \mathrm{C}$ and has the ability to withstand temperature upto $50^{\circ} \mathrm{C}$. The cell wall of conidia is made up of hydrophobin protein (expressed by well characterized gene RodA). This hydrophobin layer masks the fungal virulence factors due to which conidia are not recognized by immune cells of the host. The conidia also contain negatively charged carbohydrates such as sialic acid which helps them to adhere to the pulmonary wall containing fibronectin and laminin proteins. Besides this, the presence of melanin on the surface of conidium appears to protect it from ultraviolet light and reactive oxygen species (ROS). The ROS are used by alveolar macrophages to phagocytose the conidia. The absence of melanin in the cell wall of conidium makes it less virulent and more susceptible towards phagocytosis. The melanin component of a cell wall is synthesized from the 1,8-dihydroxynapthalene (called as DHN melanin) (Boral et al., 2018). The inner cell wall of A. fumigatus is composed of $\beta-1,3$-D-glucan to which chitin and galactosaminoglycan (GAG) are covalently attached. During germination, the conidia shed out its hydrophobin and melanin layer and swells up to form hyphae in the lungs (Van de veerdonk $\boldsymbol{e t}$ 
al., 2017). During this growth period, A. fumigatus is exposed to various stress conditions like iron acquisition, nutrient limitation, glucose deprivation and alkaline stress in host body. A. fumigatus adapt to these stress conditions by activating the expression of 'Glitoxin'. Glitoxin is a secondary metabolite produced by the fungus that shows immunosuppressive and cytocidal activity in host body. The fungus also gets benefits from the nutrients released by Glitoxinmediated killing of host cells (Kwon Chung and Sugui, 2013).

\section{RISK FACTORS}

The spectrum of diseases associated with A. fumigatus is very broad. It causes chronic pulmonary aspergillosis (CPA), acute invasive aspergillosis and allergic syndromes. The immunodeficiency of the host, which includes neutropenia, corticosteroid induced immunosuppression, hematological diseases, long term use of cytotoxic drugs like cyclophosphamide (prevents graft rejection in patients), is the main predisposing risk factor responsible for the development of invasive aspergillosis (IA). The patients undergoing hematopoietic stem cell transplant (HSCT) and solid organ transplantations (SOTs) may acquire some kind of immune defects which also contributes to the development of Aspergillus-related disease (Van de veerdonk et al., 2017). Patients suffering from severe lung disease such as chronic obstructive pulmonary disease (COPD) are at major risk to develop CPA and chronic necrotizing pulmonary aspergillosis (CNPA) that can cause fungal growth in damaged tissues. Patients having long stay in intensive care units (ICU) and those infected with tuberculosis or Human immunodeficiency virus (HIV) are also at great risk to develop aspergillosis in lungs. The allergic bronchopulmonary aspergillosis (ABPA) mainly occurs in patients having severe asthma and they immediately develop allergic response against the conidia of Aspergillus. The development of IA in patients is also due to lack of proper awareness and poor availability of microbiological tests to diagnose the infections (Garcia rubio et al., 2017)

\section{TRIAZOLE RESISTANCE IN A. FUMIGATUS}

The azole resistance has become a global health threat as spores of A. fumigatus can disperse very easily by air currents. It has been studied that $90 \%$ of the azole resistant $A$. fumigatus isolates contain TR34/L98H (Tandem repeat-TR) mutation in cyp51A. This resistant genotype is also isolated from the azole naïve patients, who were never given any azole drug treatment, but might have received sufficien azole challenge from the environment. Azole fungicides are mainly used to control the mildews and rust of grains, fruits and vegetables and powdery mildews in vines, berries, and to protect other fruit products from the attack of phytopathogenic fungi like Magnaporthe grisea or Fusarium graminearum (Snelders et al., 2009) A fumigatus is a saprophytic fungi but not a phytopathogen, so it is not the target organism of agricultural fungicides (Chowdhary et al., 2012 a,b; Snelders et al. 2012).

Among azoles, it has been seen that triazoles have better antifungal activity as compared to diazoles. There are at present only three licensed medical triazoles that are available for the treatment of Aspergillus-related diseases in clinics (itraconazole, voriconazole and posaconazole) as compared to more than twenty five types of azole fungicides that are used for agricultural purposes. These agricultural azole fungicides (difenoconazole, bromuconazole, propiconazole, tebuconazole and epoxiconazole) share similar chemical and structural properties to the medical triazoles that lead to development of cross resistance in A. fumigatus (Snelders et al., 2012; Chowdhary et al., 2013). These resistant isolates, when present in human surroundings, enter human body and lead to azole resistant fungal disease.

The triazoles are also termed as sterol demethylase inhibitors (DMI) or sterol biosynthesis inhibitors (SBI). The triazole resistance in azole resistant Aspergillus fumigatus (ARAF) can be intrinsic (primary) or acquired (secondary) in nature The intrinsic resistance is defined as the inherent mutation present in almost all the isolates of a given species to a drug without having any prior exposure to a drug. The acquired resistance to azole may develop in response to extended exposure of fungi to azoles that may exerts a strong selection pressure and results in the emergence of azole resistant isolates (Snelders et al., 2008). There are mainly two hypotheses that support the development of azole resistance in clinical isolates:

1) The patient route/ azole therapy: Treatment of patients for a longer period of time with azole drugs results in the selection of resistant mutant fungal isolates (Arendrup et al. 2010). Asexual sporulation in A. fumigatus facilitates the transfer of resistance mutation through the dispersal of spores (Chowdhary and Meis, 2018, Resendiz sharpe et al., 2018). These spores are generally formed in the lungs of patients suffering from cavitary aspergillosis. The formation of asexua spores in lungs would allow the escape of resistant uninucleate spores from the heterokaryotic mycelium that germinate and form the entire colony that expresses the resistance mutations (Zhang et al., 2015; Verweij et al., 2009b). The formation of multiple spores also enables the fungi to adapt to the selective pressure exerted by the azole drugs. However, patients suffering from acute or chronic invasive aspergillosis, do not exhibit asexual stages of spores in lungs. In these patients, infection generally proceeds through the help of invasive hyphae
Though, it is possible to develop resistance mutation in hyphae but it would have a very little effect on the phenotype of fungus because hyphae are surrounded by large number of wild type nuclei.

2) The environmental route: The isolation of azole resistant isolates from the azole naïve patients shows that they can procure azole resistant fungal infection from the environment. These isolates generally contain a tandem repeat (TR) in the promoter region along with point mutation in the coding region of cyp51A. There are mainly two reasons which justify the substantial use of azole fungicides in agriculture, first these compounds are not very expensive and secondly, they show a broad spectrum activity against various fungi. Generally all azoles have fungistatic effect rather than fungicidal effect (Azevedo et al., 2015). However azole compounds are very stable in nature and can persist in the environment for several months. Compost plays an important role in the development of azole resistance. It acts as an ecological niche for the various spores of fungi along with azole residues in it. Therefore, compost present in garden and agricultural field can act as a route for the emergence of resistant isolates (Snelders et al., 2009).

In A. fumigatus different patterns of azole resistance are identified ranging from multi azole resistance to pan azole resistance and these are found to be more common than resistance to single triazole. A. fumigatus contains two copies of target genes cyp51 (=ERG11 in yeast): cyp51A and cyp51B, but each gene encodes different structural protein. The cyp $51 A$ gene encodes lanosterol $14 \alpha$-demethylase required for ergosterol synthesis while $c y p 51 B$ is required for the alternative functions in a cell, concerned with growth. Furthermore, till now no mutation has been detected in cyp51B gene that could be credited for azole resistance (Snelders et al., 2015). The triazole class of antifungal compounds inhibits the lanosterol $14 \alpha$-demethylase enzyme. The enzyme plays a key role in the biosynthesis of ergosterol. The nitrogen atom of five membered triazole-ring binds to iron atom of heme group present in the active site of enzyme and inhibits the demethylation of lanosterol at C-14 thereby leading to the accumulation of methylated sterols in a cell. The accumulation of methylated toxic sterols in a cell makes plasma membrane leaky and impairs the growth of fungi (Bossche $\boldsymbol{e t}$ al., 1995). There are many factors which determine the emergence of resistant isolates such as population size of fungi, its mode of reproduction, concentration of antifungal used and pharmacokinetic/ pharmacodynamic (PK/PD) properties of the drug used (Buil et al., 2019). A clear understanding on emergence of triazole resistance in this fungus can be gained by understanding the basic structure of CYP51A enzyme, its role in the cell and how mutations in the cyp51A gene obliterates the azole binding to the active site of the enzyme.

\section{Structure of CYP51A Protein}

It has been studied that wild type CYP51A protein contain two ligand entry channels called as channel 1 and 2. The cyp51A sequence of Mycobacterium tuberculosis shares about $28 \%$ sequence identity with the cyp51A sequence of $A$ fumigatus (Snelders et al., 2010). The structure of wild type CYP51A protein of A. fumigatus is based on the protein homology model and X-ray crystallography structure of Mycobacterium tuberculosis protein (Chowdhary et al., 2014; Gollapudy et al., 2004; Xiao et al., 2004). The ligand entry channels helps in the binding of lipophilic sterol molecules and allow the docking of azole compounds at the active site of the protein. The presence of single nucleotide polymorphisms (SNPs) in cyp51A gene can cause structural alterations in CYP51A protein (Abdolrasouli et al., 2015). The resistance to azole class of antifungal compounds depends upon the structural properties of the protein and its interaction with the compounds. The amino acids in codons G138, G54, M220 are located at the opening of the ligand entry channels. The G138 is present at the helix of channel 1, while G54 and M220 are located at the opening of channel 2 (Figure 1). All these residues make direct interactions with the azole that are required for binding at the heme group of the protein. The channel 1 is located parallel to the plane of heme group while channel 2 is perpendicular to the plane of heme group (Snelders et al., 2010). 


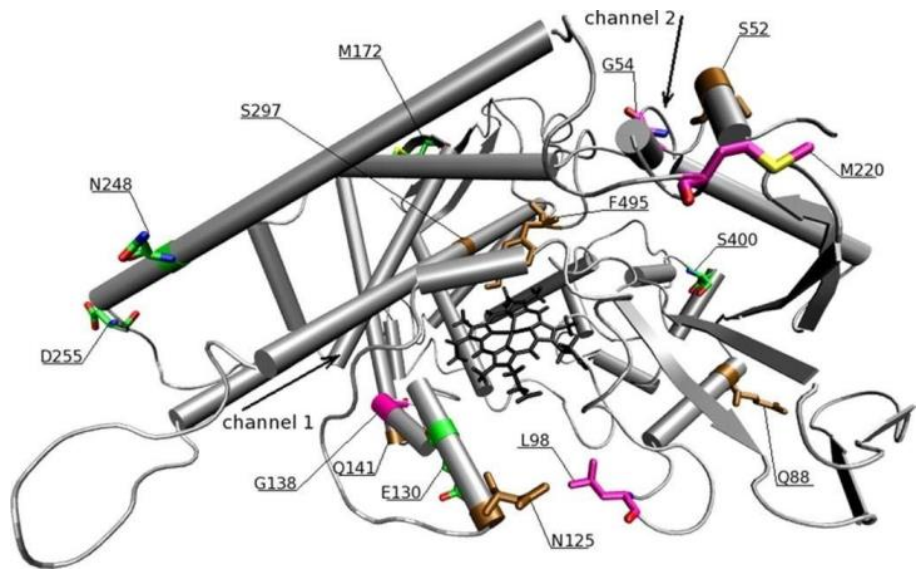

Figure 1 CYP51A protein model of A. fumigatus- This model shows the importan amino acids associated with resistance to azole compounds. The two ligand channels are indicated by arrows. The heme cofactor is represented black in color (Eveline Snelders et al. Antimicrob. Agents Chemother. 2010 doi:10.1128/AAC.01599-09) (Permission for Re-Use by ASM, License Number4978820850448)

The amino acid leucine at codon 98 in A. fumigatus is highly conserved and very important for maintaining the structural integrity of the CYP51A protein. It also plays a pivotal role in the docking of azole. The L98 residue forms an arch or gate like structure. This residue is neither located at substrate binding cleft nor at the heme group but present in a conserved loop like structure that connects two helices (B and B') in the protein structure. In wild type CYP51A protein the hydrophobic leucine residue forms an interaction with the proline 124 located on adjacent loop (BC) in the tertiary structure of the protein (Snelders et al., 2011).

\section{Point Mutation in cyp51A Gene}

Non-synonymous mutation at G54 and M220 leads to the substitution of smaller hydrophobic residues with the larger ones that will cause the blocking of channel 2 and inhibit the docking of azole compound. However there are two ligand entry channels present, but mutation in any one of the channel can inhibit the binding of azole compounds (Chowdhary et al., 2014). Majority of the mutations, mostly amino acid substitutions, are associated with channel 2 in CYP51A protein. These will cause a structural distortion in the CYP51A protein that will further inhibit the docking of azole molecules. Besides, the position and type of amino acid substitution in CYP51A protein also determines the pattern of azole cross resistance (Howard et al., 2009). Isolates having mutation at G54 residue will show cross resistance to itraconazole (ITC) and posaconazole (POS). Mutation at G54 includes 4 types of amino acid substitution and these are characterized as G54E, G54V, G54R, and G54W. The mutation G54W, for example, results in the ITC and POS resistant phenotype but it shows some susceptibility toward the voriconazole (VRC). In this type of mutation, glycine is replaced with the larger and hydrophobic tryptophan amino acid, so it will block the channel 2 and disturb the docking of azole molecule (Snelders et al., 2010). Both ITC and POS contain a long side chain and have ring like structure at the tail. Presence of two rings in the long side chains of ITC and POS will help them to bind G54 and M220 at ligand entry channel for the stable docking at heme cofactor. However, VRC is a compact molecule and lacks a long side chain; therefore it is not able to make any kind of interaction with these residues (Fraczek $\boldsymbol{e t}$ al., 2011). The absence of cross resistance between the ITC and VRC is mainly due to the presence of differences in their chemical structures (Meneau and Sanglard, 2005). Resistance to ITC drug is generally associated with reduced susceptibility to POS as well, because both these drugs have similar structures. Therefore, isolates resistant to ITC also possess elevated Minimum Inhibitory concentration (MIC) to POS (Howard et al. 2009; Xiao et al. 2004). Therefore, mutation at G54 and M220 will affect the docking of only long chain azole compound such as ITC and POS (Fraczek et al., 2011).

The second type of point mutation in CYP51A occurs at M220 residue with amino acid substitution include M220V, M220K, M220T, M220I that shows resistance to ITC with decreased susceptibility to VRC and POS (Table 1); while isolates carrying amino acid substitution G138C show pan azole resistant phenotype (Mellado et al., 2004).

The G448 residue is present at the opposite side of the heme group and it has the ability to alter the position of heme group. The G448S mutation shows resistance to voriconazole (VRC) with a reduced susceptibility to itraconazole and posaconazole (Snelders et al., 2010). This mutation acts by reducing the ability of binding of azole compound to heme group (Fraczek et al., 2011).
Table 1 Azole Resistance due to mutation in cyp51A

\begin{tabular}{|c|c|c|}
\hline $\begin{array}{l}\text { Point Mutation in } \\
\text { cyp51A at position }\end{array}$ & $\begin{array}{l}\text { Amino Acid } \\
\text { Substitution }\end{array}$ & Phenotype of the mutant \\
\hline 54 & $\begin{array}{l}\text { G54E, G54V, G54R, } \\
\text { G54W }\end{array}$ & $\begin{array}{l}\text { Resistant to ITC } \\
\text { Resistant to POS } \\
\text { Sensitive to VRC }\end{array}$ \\
\hline 220 & $\begin{array}{l}\text { M220V, M220T, } \\
\text { M220I, M220K }\end{array}$ & $\begin{array}{c}\text { Resistant to ITC } \\
\text { High MIC/Resistant to } \\
\text { POS } \\
\text { High MIC/Variable } \\
\text { resistance to VRC }\end{array}$ \\
\hline 448 & G448S & $\begin{array}{l}\text { High MIC/Resistant to } \\
\text { ITC } \\
\text { High MIC/Variable } \\
\text { resistance to POS } \\
\text { Resistant to VRC } \\
\end{array}$ \\
\hline 98 & L98H & $\begin{array}{c}\text { Multiple Azole } \\
\text { resistance }\end{array}$ \\
\hline $\begin{array}{l}46,172,248,255, \\
427\end{array}$ & $\begin{array}{c}\text { F46Y, M172V, N248K, } \\
\text { D255E, E427K }\end{array}$ & $\begin{array}{l}\text { Intermediate Azole } \\
\text { susceptibility }\end{array}$ \\
\hline
\end{tabular}

(ITC-Itraconazole, POS- Posaconazole, VRC-Voriconazole) (Garcia rubio et al., 2017; Fraczek et al., 2011)

The amino acid substitution L98H also obstructs the binding of azoles (Snelders et al., 2011). The amino acid substitution from leucine to histidine results in the change of hydrophobicity at the surface of protein, as leucine is hydrophobic in nature while histidine is a polar residue. L98 is the only hydrophobic residue present on the hydrophilic surface of CYP51A protein. This results in narrowing of diameter of channel 1 and inhibits the entry of the ligand. Additionally, mutation at $\mathrm{L} 98$ residue will re-localize the tyrosine residues at position 107 and 121 which further leads to blocking of channel 2 and result in the multiple azole resistant (MAR) phenotypes (Snelders et al., 2010, 2011). The tyrosine residue at 107 and 121 makes hydrogen bond with the two carboxyl group of the heme. The negatively charged carboxyl group will attract the histidine towards itself to neutralize negative charge, thereby blocking the entry of ligand though channel 2.

\section{Tandem Repeats in cyp51A Gene}

One of the most common resistance mechanism associated with azole compounds in both clinical and environmental isolates includes a 34 base pair tandem repeat (TR34) in the promoter region of cyp51A along with point mutation (L98H) in coding region. Changes in the promoter region of cyp51A will result in overexpression of gene (Chowdhary et al., 2017). The site directed mutagenesis studies shows that both 34 base pair tandem repeats and L98H mutation are essential for the emergence of ARAF (Snelders et al., 2011).

Another mechanism of resistance mutation includes a 46 base pair tandem repeat in promoter region of cyp $51 \mathrm{~A}$ gene along with amino acid substitution of tyrosine for phenylalanine and threonine for alanine at positions 121 and 289 respectively (TR46/Y121F/T289A). The isolates carrying TR34/L98H mutation will show ITC resistant phenotype while isolates having TR46/Y121F/T289A phenotype show resistance to VRC (Lestrade et al., 2019).

Presence of tandem repeats at the 5'end of cyp51A gene is an important mechanism for the development of azole resistance. The presence of different types of TR variants (TR34, TR46, TR53, and TR46 ${ }^{3}$ ) in isolates result in the emergence of pan azole resistant phenotypes. Over the past few years, new TR variants are reported that exhibit variation in the number (triplication in TR34 and TR46) and length of duplicated region such as TR120 (Buil et al., 2019; Hare et al., 2019).

The presence of a specific mutation in cyp51A gene also corresponds to development of specific azole resistance in fungal isolates. For example, the single nucleotide changes in cyp51A gene are thought to occur in patients that are having long term azole therapy (Lestrade et al., 2019). The tandem repeats in promoter region are mainly found in plant pathogenic molds and they generally acquire resistance mutation during the exposure of azole fungicides in environment. These azole resistant phenotypes contain mutation at the transcriptional promoter or enhancer region (Verweij et al., 2016a,b). The incorporation of tandem repeats or duplication of the region mainly occurs during the sexual reproduction of the mold (Verweij et al., 2009b).

\section{Analogous Binding of Medical Triazoles and Agricultural Fungicides to CYP51A}

The use of sterol demethylase inhibitors (DMIs) as fungicides was authorized between 1990 and 1996 whereas the emergence of first TR34/L98H fungal isolate occurred in 1997 (Snelders et al., 2012). Both clinical and environmental isolates carry this mutations and show cross resistance to medical and agricultural azoles. The agricultural use of azole fungicides is thought to be a major route responsible for the emergence and spread of TR34/L98H and TR46/Y121F/T289A mutants. 
The hydrophobic residues (Y121, F115, V120, L128) present at the active site of CYP51A protein makes vanderwal interactions with the di-halogenated phenyl group of triazoles, while the nitrogen atom of the five membered ring of triazole makes interactions with the heme group of CYP51A protein. In addition to this, the D-propionate ring $\left(\mathrm{C}_{2} \mathrm{H}_{5} \mathrm{COO}^{-}\right)$of the heme moiety forms hydrogen bond with hydroxyl group of triazoles (Chowdhary et al., 2013; Snelders et al., 2011) (Figure 2). The DMI fungicides also bind to the hydrophobic pocket created by the same residues in the target enzyme of A. fumigatus. The Y121 residue is very essential for the maintenance of the integrity and stability of heme group at the active site of a CYP51A protein. This residue forms a hydrophobic pocket and is used by all types of azole compounds (ITC, POS, VRC and DMI fungicides) for the interaction. Amino acid substitution Y121F therefore disrupts the formation of hydrogen bond between the heme group and hydroxyl group of tyrosine (Snelders et al., 2015).

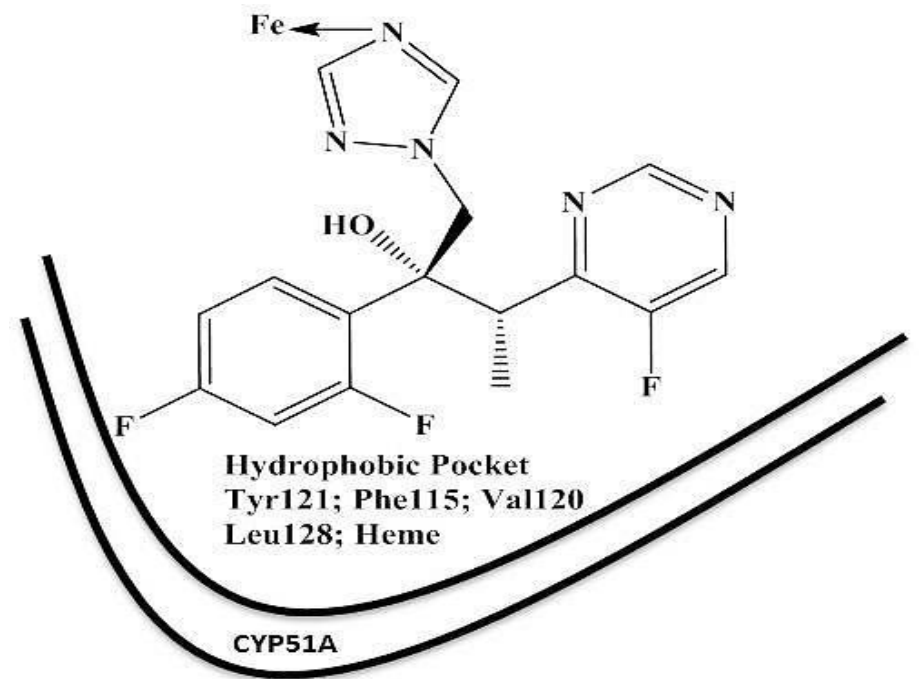

Figure 2 Binding of medical triazoles (Voriconazole) to CYP51A protein (adapted from Chowdhary et al., 2013).

\section{Non cyp51A Mutations}

Mutations that do not involve cyp51A are also reported in clinical isolates conferring azole resistance (Sharma et al., 2019). These mutations are classified into different types-a) overexpression of efflux pumps, b) upregulation of sterol regulatory element binding protein (SrbA), c) mutation in HapE and (d) mutation in $h m g 1$ gene

\section{a. Overexpression of efflux pumps}

One of the most important mechanism of resistance includes the over expression of efflux transporters that reduces the intracellular concentration of azole in the cell. Molecular characterization of the isolates shows that the A. fumigatus contain wild type cyp51A gene but still show azole resistance, which is due to the overexpression of $c d r l B$ gene encoding the $\mathrm{ABC}$ transporter (Paul et al., 2013). Overexpression of ATP binding cassette (ABC) transporters and major facilitator superfamily (MFS) transporters has been found in azole resistant isolates. The $\mathrm{ABC}$ transporter contains two membrane spanning domain and two nucleotide binding domain that catalyze the ATP hydrolysis for the drug transport. On the other hand, MFS transporters are transmembrane proteins and they utilize the electrochemical proton motive force for the drug efflux (Sanglard, 2016). $C d r 1 B$ is the only known $\mathrm{ABC}$ transporter that mediates azole resistance and $A f u M D R 3$ is a MFS transporter which is found to be overexpressed in itraconazole (ITC) resistant isolates (Slaven et al., 2002; Fraczek et al., 2013). A potential transcriptional regulator AtrR regulates the expression of gene encoding Cdr1B transporter. Mutation in this transcription factor will result in overexpression of the gene encoding $\mathrm{Cdr} 1 \mathrm{~B}$ transporter.

\section{b. Upregulation of Sterol regulatory element binding protein (SrbA)}

Sterols are defined to be the major component of the fungal cell membrane. They are required for the maintenance of fluidity and permeability of the membrane. The mammals contain cholesterol as a principal sterol in membrane while fungi contain ergosterol in its membrane. The sterol regulatory element binding proteins (SREBPs) acts as a regulator for the biosynthesis of ergosterol. It generally binds to the sterol regulatory element (SRE) present at the promoter region of the gene and controls the transcription of genes involved in biosynthesis of sterols. The $A$. fumigatus contain SREBP homolog called as SrbA. It is defined as a transcription factor that contains basic helix loop helix (bHLH) as a DNA binding domain (Gsaller et al., 2016; Dhingra and Cramer, 2017). The SrbA binds to SRE motif in the promoter region and directs the transcription of several genes such as cyp $51 A / B$ that are involved in the synthesis of ergosterol in A. fumigatus. The presence of tandem repeats in TR34 isolates results in the duplication of SRE motifs in promoter of cyp51A gene (Meis et al., 2016; Gsaller et al., 2016). Thus, binding of SrbA to SRE in TR region leads to increased expression of cyp51A gene. Therefore, SrbA plays crucial role in maintaining sterol profile of cell membrane of the fungi. The srbA null mutant shows hyper susceptibility toward the azole compounds (Willger et al., 2008).

\section{c. Mutation in HapE}

The repeat sequence in TR34 is bound by both SrbA and CCAAT binding complex $(\mathrm{CBC})$. It has been identified that CCAAT motif present in the 5 'untranslated region of cyp51A gene of A. fumigatus plays an important role in the expression of cyp51A gene. $\mathrm{CBC}$ is a multi-subunit transcription factor and contains three subunits (HapB/HapC/HapE). The $\mathrm{CBC}$ recognizes a CGAAT and CCAAT motif present in the 34-mer promoter region of cyp51A gene. It balances the action of $\mathrm{SrbA}$ and acts as a negative regulator of ergosterol biosynthesis. Inactivation of $\mathrm{CBC}$ through mutation results in the increased expression of several enzymes of the ergosterol biosynthetic pathways through transcriptional activation and confers resistance to azole compounds. Mutation in HapE subunit at P88L impairs the binding affinity of CBC at CCAAT (Camps et al., 2012a,b). Therefore, mutation in HapE subunit will results in the overexpression of sterol encoding gene by increasing the binding of SrbA (Gsaller et al., 2016).

d. Mutations in hmg1 Gene Confers Resistance to Triazole in A. fumigatus The biosynthesis of ergosterol starts with the acetyl-CoA and requires large amount of ATP for its formation. The enzyme HMG-CoA reductase catalyzes the synthesis of mevalonate from HMG-CoA. It is defined as a rate determining step in the biosynthesis of sterol in eukaryotes (Hagiwara et al., 2018). The sterol sensing domain of HMG-CoA reductase is very essential for the regulation of sterol biosynthesis. The isolate having mutation S269F, S269Y in the $h m g 1$ gene (that expresses HMG-CoA reductase) exhibits reduced susceptibility towards the azole antifungal. The mutation S269F and S269Y alter the activity of HMG-CoA reductase enzyme by diminishing its sterol sensing ability (Hagiwara et al., 2018; Rybak et al., 2019). Presence of large amount of ergosterol in a cell will require more azole drug to inhibit the growth of fungi. Thus, the content of ergosterol in the cell membrane is a primary determinant involved in development of azole resistance.

\section{ASSESSMENT OF ANTIFUNGAL DRUG RESISTANCE}

The Clinical Laboratory Standards Institute (CLSI) and the European Committee on Antimicrobial Susceptibility Testing (EUCAST) has developed standard protocols to determine antifungal susceptibility in yeasts and molds. The methods developed by them are used to determine MIC values (expressed in microgram per milliliter). CLSI and EUCAST have defined the epidemiological cut off values (ECV or ECOFF) and clinical breakpoints (based on MIC distribution, PK/PD of azoles, dose of drug, resistance mechanisms and outcome of treatment) for the study of triazole resistance in A. fumigatus (Sanglard, 2016; Verweij et al., 2009a). ECOFF values helps to identify non-wild type isolates that exhibit resistance to antifungal. They have also proposed the methods to identify in vitro resistance against the triazole in fungi. The resistant isolates generally show elevated MIC as compared to wild type isolates. However, MIC testing of various mold are not available, therefore these isolates are generally sent to the clinical mycology laboratories which can cause further delay in treatment. Therefore, therapeutic failure often occurs because patient is not able to respond to drug at a standard dose.

\section{LABORATORY METHODS TO DETECT ASPERGILLUS RELATED INFECTION}

In order to eliminate infections caused by fungi, it is very important to diagnose the infection at very early stage. Failure to detect azole resistance mutation can increase the mortality rate among the azole treated patients. To overcome these problems, scientists have developed novel diagnostic methods that are mainly used for the identification of the species involved in infection. Most of the microbiological laboratories in developing countries depend upon the conventional methods to identify Aspergillus species. The conventional method includes a direct microscopic examination, histopathological analysis, morphological character identification and standard microbiological cultures. The microscopic examination of a tissue having invasion by fungi may provide the diagnostic clue for IA. Gomori Methenamine Silver stain (GMS) and Periodic Acid-Schiff (PAS) can be used to identify IA in tissue sections (Ullmann $\boldsymbol{e t}$ al., 2018). Most of these stains are inexpensive and can be used to study various specimens such as Bronchoalveolar Lavage (BAL), Cerebrospinal fluid (CSF), sputum and tissue sections. However, these methods do not provide the accurate identification of the causative organism at species level (Arvanitis et al., 2014). The culture based methods are still used to detect triazole resistance but these methods are time consuming and have very less sensitivity. It has been studied that most of the patients suffering from aspergillosis or any other Aspergillus-related disease are found to be culture 
negative. Therefore, analysis of the resistance mutations in culture negative patients is very difficult. Thus, early diagnosis of IA and initiation of antifungal therapy may improve the chances of survival in patients. But it is very difficult to recognize IA at very early stages.

There are different types of serological tests that are available such as immunodiffusion, ELISA and western blotting. ELISA assay targets the Galactomannan (GM) antigen. GM is a polysaccharide made up of mannan backbone and contains galactofuranosyl as side chain. This antigen is released by the Aspergillus during its early stage of infection. The level of fungal markers including GM antigen and $\beta-1,3-\mathrm{D}$-glucan in the serum can be reported as optical density index (ODI). However, this assay generally fails to determine the causative organism involved in infection and its susceptibility towards antifungal (Ullmann et al., 2018).

Many assays are available for the antifungal susceptibility testing such as disk diffusion assay, Etest, MIC strip kits, Sensititre YeastOne colorimetric assay and MTT assays. It is always suggested to perform an antifungal susceptibility test whenever the patient is tested positive for the A. fumigatus culture. The problem with this approach is that MIC determination is not routinely done in microbiological laboratories. Recently, the matrix assisted laser desorption ionization time of flight mass spectrometry (MALDI-TOF-MS) has improved the chances for the diagnosis of aspergillosis (Masih et al., 2016). It provides rapid and accurate identification of the isolates. MALDI-TOF-MS is based on mass spectrometry and identifies the protein fingerprints of the microorganisms (Cassagne et al., 2016). It is possible to identify organism at species, genus and even strain level by comparing the spectral data of causative organisms with the reference spectra provided at the database in computer software (Arvanitis et al. 2014)

\section{MOLECULAR METHODS TO DIAGNOSE AZOLE RESISTANCE IN $A$ FUMIGATUS}

Invasive fungal infections (IFIs) constitute a serious threat to immunocompromised patients. Species identification is a first step to detect the resistance mutation as it will help in the study of intrinsically resistant species (Lestrade et al., 2019; Ullmann et al., 2018). High mortality rate associated with the azole resistant isolates underlies the need of non-culture based methods to detect the species involved in infection and triazole resistance in clinical samples (Jenks et al., 2019; Buil et al., 2018). The molecular method detects the resistance mutation in CYP51A protein and ensures the amplification of only fungal DNA and avoid any kind of cross reactivity with human DNA. A multiplex real-time PCR assays are available that detects one (TR34/L98H; Mycogenie developed by Ademtech, Pessac, France) or two (TR34/L98H and TR46/Y121F/T298A; AsperGenius, developed by PathoNostics, Maastricht, Netherlands) resistance mutation that are associated with the environmental route of transmission of azole resistance (Rath and Steinmann, 2018). Both these assays have advantages and disadvantages. The major benefit of AsperGenius assay includes its ability to detect the mixed infections and resistance mutation in culture negative patients The AsperGenius assay detects mutation by the help of melting curve analysis (Meis et al., 2016; White et al., 2015). This assay shows its diagnostic effect in BAL fluid and serum samples while MycoGenie PCR system is generally recommended for the testing of biopsy, respiratory and serum samples. MycoGenie PCR system shows a very high sensitivity to detect the 28S rRNA gene of $A$ fumigatus (Jenks et al., 2019; Rath and Steinmann, 2018). The important drawback includes the low sensitivity of the assays because cyp51A gene is a single copy gene present in each Aspergillus cell so that detection of resistance markers in serum might not be successful (Verweij et al., 2015). The problem associated with these commercial test kits is that they are only able to identify TR associated mutations. Therefore a negative test by these methods does not mean that the patient is not suffering from azole resistant aspergillosis (Lestrade et al., 2019, Buil et al., 2019; Meis et al., 2016).

Microsatellite analysis is a best approach to study the genetic diversity between the fungal isolates. The next generation sequencing techniques such as illumina sequencing, amplicon sequencing of ITS regions or ribosomal gene, helps in determining the severity of fungal infections. The amplicon sequencing of ITS regions and whole genome sequencing of fungal isolates helps in identifying the species involved in infection (Zoll et al., 2016).

Presence of point mutations in the cyp51A gene is considered to be a main mechanism for the emergence of azole resistant isolates. Molecular approaches that targets the mutation associated with the coding region of cyp51A gene (G54, M220 G138, G448) are still lagging behind. Pyrosequencing is an important DNA sequencing technique based on 'sequencing by synthesis' principle that can rapidly screen out point mutations associated with the azole resistance (Van der torre $\boldsymbol{e t}$ al., 2020).

Nucleic acid amplification provides a rapid, sensitive and simple technique that can be used in clinical laboratories to identify early stage infections. Most of the PCR based techniques relies on the use of specific primer targeting the internal transcribed sequence (ITS) of the rDNA which helps in identifying the species. Other gene loci can also be useful to study the Aspergillus species such as $\beta$-tubulin (benA) and calmodulin ( $c a M)$ gene that can act as a secondary identification markers for the identification of causative organism involved in infection. Taxonomic tool such as ribosome gene and inter transcribed spacer regions are generally used for inter section level identification in A. fumigatus while the $\beta$ tubulin locus is used for the identification of individual species belonging to various Aspergillus sections (Chowdhary et al., 2016; Zakaria et al., 2020).

\section{EPIDEMIOLOGY OF CLINICALLY ISOLATED ASPERGILLUS FUNGI}

As per the epidemiological data, A. fumigatus is the predominant etiological agen isolated from the immunocompromised patients. Prospective cohort studies of transplant-associated fungal infections in the United States carried out by Transplant-Associated Infection Surveillance Network (TRANSNET) have also reported that $A$. fumigatus is the leading cause of invasive aspergillosis followed by A. flavus, A. niger, A. terreus (Kontoyiannis et al., 2010). By analyzing this data, it has been realized that non-fumigatus species can also be pathogenic in humans and animals. Recently, cryptic species of Aspergilli had received much attention due to development of new molecular tools for identification of species. Species that cannot be distinguished morphologically from the leading pathogen of their section are called as 'cryptic' species. Within the section Nigri, A. niger and $A$. tubingensis is the most common causative agent of otomycosis, pulmonary aspergillosis and aspergilloma. Aspergillus section Fumigati contains more than 60 species, out of which 15 species are reported in clinical specimens (Pappas et al., 2010). It has been reported that A. lentulus, A. viridinutans, A. fumigatiaffinis A. fumisynnematus, A. pseudofischeri, A. hiratsukae, A. udagawae are frequently misidentified as A. fumigatus. The cryptic species in section Nigri and section Fumigati show different antifungal susceptibility and different level of pathogenicity from those of $A$. fumigatus. The growing incidence of azole resistant cryptic species demands the accurate identification and antifungal susceptibility of the isolates (Pappas et al., 2010; Hagiwara et al., 2016)

\section{TREATMENT OPTIONS}

It has been successfully demonstrated that azole resistance is the predominant cause of treatment failure in most cases. The elevated MIC shown by azole resistant isolates reduces the efficiency of azole monotherapy in treatment (Lepak et al., 2013). CD101 (biafungin) is a novel echinocandin that has been found to be effective in mouse model suffering from IA. ITC is mainly used in the treatment of CPA while VRC is used for IA. Amphotericin B (AmB) is a polyene and shows very poor oral absorption therefore it is solubilized with deoxycholate for the intravenous administration. AmB-deoxycholate is a potent antifungal drug with a broad spectrum activity, but it shows nephrotoxicity and infusion reactions (Stone et al., 2016). These disadvantages promoted the development of less toxic lipid based formulations of AmB such as amphotericin B lipid complex (ABLC). The new formulation includes $\mathrm{AmB}$ nanoparticle suspensions and $\mathrm{AmB}$ arabinogalactan or AmB-PEG (AmB-Polyethylene glycol) that shows high efficacy with little toxicity. The primary mechanism of AmB action includes its ability to form ion channels in the plasma membrane leading to leakage of ions from the cell. The second mechanism of action involves the oxidative damage of a cell by producing ROS. Lipid based formulation are generally recommended to avoid any kind of $\mathrm{AmB}$ related nephrotoxicity. The available lipid formulations are ABLC (Abelcet), AmB colloidal dispersion (ABCD; amphocil, Amphotec) and liposomal AmB (AmBisome) (Patterson et al., 2016).

Echinocandins are lipopeptide antifungal agent. They inhibit the synthesis of $\beta$ $(1,3)$-D-glucan, component of cell wall in fungi. Isavuconazole (ISAZ) and its azonium sulfate derivative are used as oral formulation in the treatment of IA

Another new promising agent, arylamidine T-2307 (Toyoma Chemical Company, Tokyo, Japan) targets the mitochondria of fungi and results in the loss of mitochondrial membrane potential. A new agent E121010/APX001 (Amplyx Pharmaceuticals, CA, USA) shows anti-Aspergillus activity by inhibiting the glycosyl-phosphatidylinositol dependent anchorage in the cell wall of fungi. A novel antifungal ASP2397 (Astellas Pharma, Tokyo, Japan) inhibits the fungal siderophore transport. F901318 developed by F2G Ltd (Manchester, UK), mainly inhibits the enzyme dihydroorotate (DHODH/URA1) that participates in the biosynthesis of pyrimidines (Wiederhold, 2017) in the fungi.

The natural products can also show anti-Aspergillus activity. The psoriatic skin lesion infected by fungi contains a skin associated antifungal psoriasin. It is a small sized fungicidal protein and it promotes death of fungi by chelating the intracellular zinc ions (Osherov and Kontoyiannis, 2016).

\section{HSP90 INHIBITOR, A NOVEL ANTIFUNGAL TARGETED AGAINST $A$ FUMIGATUS}

Hsp90 is a conserved molecular chaperone involved in a complex network of calcineurin signaling pathways. The calcineurin pathway is a compensatory signaling repair pathway that helps to sustain cell damage that occurs in response to antifungal. Inhibition of $\mathrm{Hsp} 90$ and calcineurin signaling pathway represents a novel approach for the increased action of antifungals. Hsp90 maintains cell integrity by preventing the misfolding and aggregation of various proteins in a cell. Hsp90 contains three structural and functional domains, the $\mathrm{N}$ terminal domain 
contain the binding site for ATP followed by a middle domain and C-termina domain that contain binding site for the various downstream effector proteins and co-chaperons (Lamoth et al., 2014). The Hsp90 inhibitor geldanamycin and its derivatives 17-allylamino-17demethoxygeldanamycin (17-AAG) and 17dimethylaminoethylamino-17-demethoxygeldanamycin (17-DMAG) shows in vitro antifungal activity against the A. fumigatus. Geldanamycin restricts the paradoxical effect of caspofungin and potentiates its antifungal activity. The echinocandin (caspofungin, anidulafungin and micafungin) class of antifungal inhibits the synthesis of $\beta-1,3-D-G l u c a n$. The paradoxical effect refers to the decrease of echinocandin activity at higher concentrations. This effect occurs in $A$ fumigatus due to the activation of calcineurin signaling pathways which increases the chitin in cell wall. Hsp90 is one of the client proteins that activate this pathway. Therefore, use of Hsp90 inhibitors such as geldanamycin and its derivatives can attenuates the paradoxical effect. Targeting of Hsp90 and calcineurin signaling pathways can result in increased susceptibility towards the antifungal.

The anti-calcineurin drug FK506 (tacrolimus and cyclosporin), Hsp90 inhibitor (Geldanamycin and its derivatives 17-AAG, 17-DMAG) and histone deacetylase (HDAC) inhibitor such as Trichostatin A exhibit in vitro antifungal activity agains various mold species. Trichostatin A inhibitor further shows synergistic interactions with caspofungin (Lamoth et al., 2015a,b).

\section{CONCLUSION}

The emergence of azole resistance in A. fumigatus is a major global health issue The article highlights importance of fungal cyp51A gene and the associated mutations in the gene that form the primary cause of emergence of azole resistant A. fumigatus mutants. The fact that these strains show cross-resistance to the three important medical triazoles and also to agricultural triazole fungicides, pointed to the spread of resistant isolates from environment to the clinics. There is therefore growing need for the development of novel approach methods to identify resistance mutations in order to initiate appropriate antifungal therapy in patients. Several new antifungals need to be developed that will show more advantage as compared to current drugs in terms of overcoming drug resistance and could offer less toxic effects. Environmental survey should be conducted to determine the prevalence of resistant isolates in agricultural fields. Furthermore, the indiscriminate usage of fungicides should be avoided in agricultural fields to minimize the emergence of resistant isolates. The increasing number of affected patients emphasizes the need for an elaborate study of fungal epidemiology in different countries and a thorough assessment of azole resistant mutations and its associated treatment failure. The future research should focus mainly on effectiveness of new pharmaceutical agents that can be used in alone or synergistically to avoid the emergence of resistant isolates.

Authors' contributions statement: The authors declare that there are no competing interests. The article was conceived, written and reviewed by $\mathrm{KW}$ and $\mathrm{HK}$

Acknowledgement: The authors express gratitude to Principal, Ramjas College, University of Delhi, for his constant encouragement. KW also wishes to acknowledge University Grants Commission (UGC) for financial support in the form of Junior Research fellowships.

\section{REFERENCES}

Abdolrasouli, A., Rhodes, J., Beale, M. A., Hagen, F., Rogers, T. R., Chowdhary, A., ... \& Fisher, M. C. (2015). Genomic context of azole resistance mutations in Aspergillus fumigatus determined using whole-genome sequencing. MBio, 6(3) http://dx.doi.org/10.1128/mBio.00536-15

Arendrup, M. C., Mavridou, E., Mortensen, K. L., Snelders, E., Frimodt-Møller, N., Khan, H., ... \& Verweij, P. E. (2010). Development of azole resistance in Aspergillus fumigatus during azole therapy associated with change in virulence. PLoS One, 5(4),

e10080.

http://dx.doi.org/10.1371/journal.pone.0010080

Arvanitis, M., Anagnostou, T., Fuchs, B. B., Caliendo, A. M., \& Mylonakis, E. (2014). Molecular and nonmolecular diagnostic methods for invasive fungal infections. Clinical microbiology reviews, 27(3), 490-526. http://dx.doi.org/10.1128/CMR.00091-13

Azevedo, M. M., Faria-Ramos, I., Cruz, L. C., Pina-Vaz, C., \& Goncalves Rodrigues, A. (2015). Genesis of azole antifungal resistance from agriculture to clinical settings. Journal of agricultural and food chemistry, 63(34), 7463-7468. http://dx.doi.org/10.1021/acs.jafc.5b02728

Boral, H., Metin, B., Döğen, A., Seyedmousavi, S., \& Ilkit, M. (2018). Overview of selected virulence attributes in Aspergillus fumigatus, Candida albicans, Cryptococcus neoformans, Trichophyton rubrum, and Exophiala dermatitidis. Fungal Genetics and Biology, 111, 92-107. http://dx.doi.org/10.1016/j.fgb.2017.10.008

Bossche, H. V., Koymans, L., \& Moereels, H. (1995). P450 inhibitors of use in medical treatment: focus on mechanisms of action. Pharmacology \& therapeutics, 67(1), 79-100. http://dx.doi.org/10.1016/0163-7258(95)00011-5
Buil, J. B., Hare, R. K., Zwaan, B. J., Arendrup, M. C., Melchers, W. J., \& Verweij, P. E. (2019). The fading boundaries between patient and environmental routes of triazole resistance selection in Aspergillus fumigatus. PLoS pathogens, 15(8), e1007858. http://dx.doi.org/10.1371/journal.ppat.1007858

Buil, J. B., Zoll, J., Verweij, P. E., \& Melchers, W. J. (2018). Molecular detection of azole-resistant Aspergillus fumigatus in clinical samples. Frontiers in microbiology, 9, 515. http://dx.doi.org/10.3389/fmicb.2018.00515

Camps, S. M., Dutilh, B. E., Arendrup, M. C., Rijs, A. J., Snelders, E., Huynen, M. A., ... \& Melchers, W. J. (2012a). Discovery of a HapE mutation that causes azole resistance in Aspergillus fumigatus through whole genome sequencing and sexual crossing. PLoS One, 7(11), e50034 http://dx.doi.org/10.1371/journal.pone.0050034

Camps, S. M., van der Linden, J. W., Li, Y., Kuijper, E. J., van Dissel, J. T., Verweij, P. E., \& Melchers, W. J. (2012b). Rapid induction of multiple resistance mechanisms in Aspergillus fumigatus during azole therapy: a case study and review of the literature. Antimicrobial agents and chemotherapy, 56(1), 10-16 http://dx.doi.org/10.1128/AAC.05088-11

Cassagne, C., Normand, A. C., L'Ollivier, C., Ranque, S., \& Piarroux, R. (2016) Performance of MALDI-TOF MS platforms for fungal identification. Mycoses, 59(11), 678-690. http://dx.doi.org/10.1111/myc.12506.

Chowdhary, A., \& Meis, J. F. (2018). Emergence of azole resistant Aspergillus fumigatus and One Health: time to implement environmental stewardship. Environmental microbiology, 20(4),

1299-1301. http://dx.doi.org/10.1111/1462-2920.14055

Chowdhary, A., Kathuria, S., Randhawa, H. S., Gaur, S. N., Klaassen, C. H., \& Meis, J. F. (2012a. Isolation of multiple-triazole-resistant Aspergillus fumigatus strains carrying the TR/L98H mutations in the cyp51A gene in India. Journal of antimicrobial chemotherapy, 67(2), 362-366. http://dx.doi.org/10.1093/jac/dkr443 Chowdhary, A., Kathuria, S., Xu, J., \& Meis, J. F. (2013). Emergence of azoleresistant Aspergillus fumigatus strains due to agricultural azole use creates an increasing threat to human health. PLoS pathog,9(10), e1003633. http://dx.doi.org/10.1371/journal.ppat.1003633

Chowdhary, A., Kathuria, S., Xu, J., Sharma, C., Sundar, G., Singh, P. K., ... \& Meis, J. F. (2012b). Clonal expansion and emergence of environmental multipletriazole-resistant Aspergillus fumigatus strains carrying the TR 34/L98H mutations in the cyp 51A gene in India. PloS one, 7(12), e52871. https://doi.org/10.1371/journal.pone.0052871

Chowdhary, A., Masih, A., \& Sharma, C. (2016). Azole resistance in moldsapproach to detection in a clinical laboratory. Current Fungal Infection Reports, 10(3), 96-106. http://dx.doi.org/10.1007/s12281-016-0265-2

Chowdhary, A., Sharma, C., \& Meis, J. F. (2017). Azole-resistant aspergillosis: epidemiology, molecular mechanisms, and treatment. The Journal of infectious diseases, 216(suppl_3), S436-S444. http://dx.doi.org/10.1093/infdis/jix210

Chowdhary, A., Sharma, C., Hagen, F., \& Meis, J. F. (2014). Exploring azole antifungal drug resistance in Aspergillus fumigatus with special reference to resistance mechanisms. Future microbiology, 9(5), 697-711. http://dx.doi.org/10.2217/fmb.14.27

Chowdhary, A., Sharma, C., Kathuria, S., Hagen, F., \& Meis, J. F. (2015) Prevalence and mechanism of triazole resistance in Aspergillus fumigatus in a referral chest hospital in Delhi, India and an update of the situation in Asia. Frontiers in microbiology, 6, 428. https://doi.org/10.3389/fmicb.2015.00428 Dagenais, T. R., \& Keller, N. P. (2009). Pathogenesis of Aspergillus fumigatus in invasive aspergillosis. Clinical microbiology reviews, 22(3), 447-465. http://dx.doi.org/10.1128/CMR.00055-08

Dhingra, S., \& Cramer, R. A. (2017). Regulation of sterol biosynthesis in the human fungal pathogen Aspergillus fumigatus: opportunities for therapeutic development. Frontiers in microbiology, 8 ,

http://dx.doi.org/10.3389/fmicb.2017.00092

Fraczek, M.G., Bromley, M., \& Bowyer, P. (2011). An improved model of the Aspergillus fumigatus CYP51A protein. Antimicrobial agents and chemotherapy, 55(5), 2483-2486. https://doi.org/10.1128/AAC.01651-10

Fraczek, M.G., Bromley, M., Buied, A., Moore, C. B., Rajendran, R., Rautemaa, R., ... \& Bowyer, P. (2013). The cdr1B efflux transporter is associated with noncyp51a-mediated itraconazole resistance in Aspergillus fumigatus. Journal of Antimicrobial Chemotherapy, 68(7), 1486-1496. https://doi.org/10.1093/jac/dkt075

Garcia-Rubio, R., Cuenca-Estrella, M., \& Mellado, E. (2017). Triazole resistance in Aspergillus species: an emerging problem. Drugs, 77(6), 599-613. http://dx.doi.org/10.1007/s40265-017-0714-4

Gollapudy, R., Ajmani, S., \& Kulkarni, S. A. (2004). Modeling and interactions of Aspergillus fumigatus lanosterol 14- $\alpha$ demethylaseA'with azole antifungals. Bioorganic \& medicinal chemistry, 12(11), 2937-2950. http://dx.doi.org/10.1016/j.bmc.2004.03.034

Gsaller, F., Hortschansky, P., Furukawa, T., Carr, P. D., Rash, B., Capilla, J., ... \& Brakhage, A. A. (2016). Sterol biosynthesis and azole tolerance is governed by the opposing actions of SrbA and the CCAAT binding complex. PLoS pathogens, 12(7), e1005775. https://doi.org/10.1371/journal.ppat.1005775

Hagiwara, D., Arai, T., Takahashi, H., Kusuya, Y., Watanabe, A., \& Kamei, K (2018). Non-cyp51A azole-resistant Aspergillus fumigatus isolates with mutation 
in HMG-CoA reductase. Emerging infectious diseases, 24(10), 1889 http://dx.doi.org/10.320/eid2410.180730

Hagiwara, D., Watanabe, A., Kamei, K., \& Goldman, G. H. (2016). Epidemiological and genomic landscape of azole resistance mechanisms in

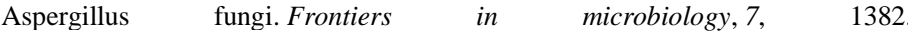
https://doi.org/10.3389/fmicb.2016.01382

Hare, R. K., Gertsen, J. B., Astvad, K. M., Degn, K. B., Løkke, A., Stegger, M., .. $\&$ Arendrup, M. C. (2019). In vivo selection of a unique tandem repeat mediated azole resistance mechanism (TR120) in Aspergillus fumigatus cyp51A, Denmark. Emerging infectious diseases, 25(3), https://doi.org/10.3201/eid2503.180297

Howard, S. J., Cerar, D., Anderson, M. J., Albarrag, A., Fisher, M. C., Pasqualotto, A. C., ... \& Denning, D. W. (2009). Frequency and evolution of azole resistance in Aspergillus fumigatus associated with treatment failure. Emerging infectious diseases, 15(7), 1068. http://dx.doi.org/10.3201/eid1507.090043

Jenks, J. D., Spiess, B., Buchheidt, D., \& Hoenigl, M. (2019). (New) Methods for Detection of Aspergillus fumigatus Resistance in Clinical Samples. Current fungal infection reports, 13(3), 129-136. https://doi.org/10.1007/s12281-019-00342-w Kontoyiannis, D. P., Marr, K. A., Park, B. J., Alexander, B. D., Anaissie, E. J., Walsh, T. J., ... \& Brumble, L. M. (2010). Prospective surveillance for invasive fungal infections in hematopoietic stem cell transplant recipients, 2001-2006: overview of the Transplant-Associated Infection Surveillance Network (TRANSNET) Database. Clinical Infectious Diseases, 50(8), 1091-1100. https://doi.org/10.1086/651263

Kwon-Chung, K. J., \& Sugui, J. A. (2013). Aspergillus fumigatus-what makes the species a ubiquitous human fungal pathogen?. PLoS Pathog, 9(12), e1003743. https://doi.org/10.1371/journal.ppat.1003743

Lamoth, F., Alexander, B. D., Juvvadi, P. R., \& Steinbach, W. J. (2015a) Antifungal activity of compounds targeting the Hsp90-calcineurin pathway against various mold species. Journal of Antimicrobial Chemotherapy, 70(5), 1408-1411. http://dx.doi.org/10.1093/jac/dku549

Lamoth, F., Juvvadi, P. R., \& Steinbach, W. J. (2015b). Histone deacetylase inhibition as an alternative strategy against invasive aspergillosis. Frontiers in microbiology, 6, 96. http://dx.doi.org/10.3389/fmicb.2015.00096

Lamoth, F., Juvvadi, P. R., Gehrke, C., Asfaw, Y. G., \& Steinbach, W. J. (2014) Transcriptional activation of heat shock protein 90 mediated via a proximal promoter region as trigger of caspofungin resistance in Aspergillus fumigatus. The Journal of infectious diseases, 209(3), 473 481.https://doi.org/10.1093/infdis/jit530

Lepak, A. J., Marchillo, K., VanHecker, J., \& Andes, D. R. (2013). Impact of in vivo triazole and echinocandin combination therapy for invasive pulmonary aspergillosis: enhanced efficacy against Cyp51 mutant isolates. Antimicrobial agents and chemotherapy, 57(11), 5438 5447.https://doi.org/10.1128/AAC.00833-13

Lestrade, P. P. A., Meis, J. F., Melchers, W. J. G., \& Verweij, P. E. (2019). Triazole resistance in Aspergillus fumigatus: recent insights and challenges for patient management. Clinical Microbiology and Infection, 25(7), 799-806. http://dx.doi.org/10.1016/j.cmi.2018.11.027

Masih, A., Singh, P. K., Kathuria, S., Agarwal, K., Meis, J. F., \& Chowdhary, A (2016). Identification by molecular methods and matrix-assisted laser desorption ionization-time of flight mass spectrometry and antifungal susceptibility profiles of clinically significant rare Aspergillus species in a referral chest hospital in Delhi, India. Journal of Clinical Microbiology, 54(9), 2354-2364. https://doi.org/10.1128/JCM.00962-16

Meis, J. F., Chowdhary, A., Rhodes, J. L., Fisher, M. C., \& Verweij, P. E. (2016).Clinical implications of globally emerging azole resistance in Aspergillus fumigatus. Philosophical Transactions of the Royal Society B: Biological Sciences, 371(1709), 20150460. https://doi.org/10.1098/rstb.2015.0460

Mellado, E., Garcia-Effron, G., Alcazar-Fuoli, L., Cuenca-Estrella, M., \& Rodriguez-Tudela, J. L. (2004). Substitutions at methionine 220 in the $14 \alpha$-sterol demethylase (Cyp51A) of Aspergillus fumigatus are responsible for resistance in vitro to azole antifungal drugs. Antimicrobial Agents and Chemotherapy, 48(7), 2747-2750. http://dx.doi.org/10.1128/AAC.48.7.2747-2750.2004

Meneau, I., \& Sanglard, D. (2005). Azole and fungicide resistance in clinical and environmental Aspergillus fumigatus isolates. Medical mycology, 43(Supplement_1), https://doi.org/10.1080/13693780500090826

Osherov, N., \& Kontoyiannis, D. P. (2016). The anti-Aspergillus drug pipeline: is the glass half full or empty?. Sabouraudia, 55(1), 118-124. http://dx.doi.org/10.1093/mmymyw060

Pappas, P. G., Alexander, B. D., Andes, D. R., Hadley, S., Kauffman, C. A., Freifeld, A., ... \& Kontoyiannis, D. P. (2010). Invasive fungal infections among organ transplant recipients: results of the Transplant-Associated Infection Surveillance Network (TRANSNET). Clinical Infectious Diseases, 50(8), 1101 1111. https://doi.org/10.1086/651262

Patterson, T. F., Thompson III, G. R., Denning, D. W., Fishman, J. A., Hadley, S., Herbrecht, R., ... \& Segal, B. H. (2016). Practice guidelines for the diagnosis and management of aspergillosis: 2016 update by the Infectious Diseases Society of
America. Clinical Infectious

Diseases, 63(4),

e1-e60 http://dx.doi.org/10.1093/cid/ciw326

Paul, S., Diekema, D., \& Moye-Rowley, W. S. (2013). Contributions of Aspergillus fumigatus ATP-binding cassette transporter proteins to drug resistance and virulence. Eukaryotic cell, 12(12), 1619-1628. https://doi.org/10.1128/EC.00171-13

Rath, P. M., \& Steinmann, J. (2018). Overview of commercially available PCR assays for the detection of Aspergillus spp. DNA in patient samples. Frontiers in microbiology, 9, 740. https://doi.org/10.3389/fmicb.2018.00740

Resendiz Sharpe, A., Lagrou, K., Meis, J. F., Chowdhary, A., Lockhart, S. R. Verweij, P. E., \& ISHAM/ECMM Aspergillus Resistance Surveillance working group. (2018). Triazole resistance surveillance in Aspergillus fumigatus. Medical Mycology, 56(suppl_1), S83-S92. https://doi.org/10.1093/mmy/myx144

Rybak, J. M., Ge, W., Wiederhold, N. P., Parker, J. E., Kelly, S. L., Rogers, P. D. \& Fortwendel, J. R. (2019). Mutations in hmg1, challenging the paradigm of clinical triazole resistance in Aspergillus fumigatus. MBio, 10(2) http://dx.doi.org/10.1128/mbio.00437-19

Sanglard, D. (2016). Emerging threats in antifungal-resistant fungal pathogens. Frontiers in medicine, 3, 11. https://doi.org/10.3389/fmed.2016.00011 Sharma, C., Nelson-Sathi, S., Singh, A., Pillai, M. R., \& Chowdhary, A. (2019) Genomic perspective of triazole resistance in clinical and environmental Aspergillus fumigatus isolates without cyp51A mutations. Fungal genetics and biology, 132, 103265. http://dx.doi.org/10.1016/j.fgb.2019.103265

Slaven, J. W., Anderson, M. J., Sanglard, D., Dixon, G. K., Bille, J., Roberts, I. S., \& Denning, D. W. (2002). Increased expression of a novel Aspergillus fumigatus $\mathrm{ABC}$ transporter gene, atrF, in the presence of itraconazole in an itraconazole resistant clinical isolate. Fungal Genetics and Biology, 36(3), 199-206. https://doi.org/10.1016/s1087-1845(02)00016-6

Snelders, E., Camps, S. M., Karawajczyk, A., Rijs, A. J., Zoll, J., Verweij, P. E. \& Melchers, W. J. (2015). Genotype-phenotype complexity of the TR46/Y121F/T289A cyp51A azole resistance mechanism in Aspergillus $\begin{array}{llll}\text { fumigatus. Fungal } \quad \text { Genetics } & \text { Biology, 82, }\end{array}$ https://doi.org/10.1016/j.fgb.2015.06.001

Snelders, E., Camps, S. M., Karawajczyk, A., Schaftenaar, G., Kema, G. H., Van der Lee, H. A., ... \& Verweij, P. E. (2012). Triazole fungicides can induce crossresistance to medical triazoles in Aspergillus fumigatus. PloS one, 7(3), e31801 http://dx.doi.org/10.1371/journal.pone.0031801

Snelders, E., Karawajczyk, A., Schaftenaar, G., Verweij, P. E., \& Melchers, W. J. (2010). Azole resistance profile of amino acid changes in Aspergillus fumigatus CYP51A based on protein homology modeling. Antimicrobial agents and chemotherapy, 54(6), 2425-2430. https://doi.org/10.1128/AAC.01599-09

Snelders, E., Karawajczyk, A., Verhoeven, R. J., Venselaar, H., Schaftenaar, G., Verweij, P. E., \& Melchers, W. J. (2011). The structure-function relationship of the Aspergillus fumigatus cyp51A L98H conversion by site-directed mutagenesis: the mechanism of $\mathrm{L} 98 \mathrm{H}$ azole resistance. Fungal Genetics and Biology, 48(11) 1062-1070. http://dx.doi.org/10.1016/j.fgb.2011.08.002

Snelders, E., Rijs, A. J., Kema, G. H., Melchers, W. J., \& Verweij, P. E. (2009) Possible environmental origin of resistance of Aspergillus fumigatus to medical triazoles. Applied and environmental microbiology,75(12), 4053-4057. http://dx.doi.org/10.1128/AEM.00231-09

Snelders, E., Van Der Lee, H. A., Kuijpers, J., Rijs, A. J. M., Varga, J., Samson, R. A., ... \& Verweij, P. E. (2008). Emergence of azole resistance in Aspergillus fumigatus and spread of a single resistance mechanism. PLoS Med, 5(11), e219. http://dx.doi.org/10.1371/journal.pmed.0050219

Stone, N. R., Bicanic, T., Salim, R., \& Hope, W. (2016). Liposomal amphotericin $\mathrm{B}($ AmBisome $\mathbb{R})$ : a review of the pharmacokinetics, pharmacodynamics, clinical experience and future directions. Drugs, 76(4), 485-500. http://dx.doi.org/10.1007/s40265-016-0538-7

Ullmann, A. J., Aguado, J. M., Arikan-Akdagli, S., Denning, D. W., Groll, A. H. Lagrou, K., ... \& Warris, A. (2018). Diagnosis and management of Aspergillus diseases: executive summary of the 2017 ESCMID-ECMM-ERS guideline. Clinical Microbiology and Infection, 24, e1-e38. https://doi.org/10.1016/j.cmi.2018.01.002

Van De Veerdonk, F. L., Gresnigt, M. S., Romani, L., Netea, M. G., \& Latge, J. P. (2017). Aspergillus fumigatus morphology and dynamic host interactions. Nature Reviews Microbiology, 15(11), 661. https://doi.org/10.1038/nrmicro.2017.90 Van der Torre, M. H., Novak-Frazer, L., \& Rautemaa-Richardson, R. (2020) Detecting azole-antifungal resistance in Aspergillus fumigatus by pyrosequencing. Journal of Fungi, 6(1), 12. https://doi.org/10.3390/jof6010012

Verweij, P. E., Ananda-Rajah, M., Andes, D., Arendrup, M. C., Brüggemann, R. J., Chowdhary, A., ... \& Kullberg, B. J. (2015). International expert opinion on the management of infection caused by azole-resistant Aspergillus fumigatus. Drug Resistance Updates, 21, 30-40. https://doi.org/10.1016/j.drup.2015.08.001

Verweij, P. E., Chowdhary, A., Melchers, W. J., \& Meis, J. F. (2016a). Azole resistance in Aspergillus fumigatus: can we retain the clinical use of mold-active antifungal azoles?. Clinical Infectious Diseases, 62(3), 362-368. http://dx.doi.org/10.1093/cid/civ885 
Verweij, P. E., Howard, S. J., Melchers, W. J., \& Denning, D. W. (2009a). Azoleresistance in Aspergillus: proposed nomenclature and breakpoints. Drug Resistance Updates, 12(6), 141-147. https://doi.org/10.1016/j.drup.2009.09.002 Verweij, P. E., Snelders, E., Kema, G. H., Mellado, E., \& Melchers, W. J. (2009b) Azole resistance in Aspergillus fumigatus: a side-effect of environmental fungicide use?. The Lancet infectious diseases, 9(12), 789-795. https://doi.org/10.1016/S1473-3099(09)70265-8

Verweij, P. E., Zhang, J., Debets, A. J., Meis, J. F., van de Veerdonk, F. L., Schoustra, S. E., ... \& Melchers, W. J. (2016b). In-host adaptation and acquired triazole resistance in Aspergillus fumigatus: a dilemma for clinical management. The Lancet Infectious Diseases, 16(11), e251e260.http://doi.org/10.1016/S1473-3099(16)30138-4

White, P. L., Posso, R. B., \& Barnes, R. A. (2015). Analytical and clinical evaluation of the PathoNostics AsperGenius assay for detection of invasive aspergillosis and resistance to azole antifungal drugs during testing of serum samples. Journal of clinical microbiology,53(7), 2115-2121. https://doi.org/10.1128/JCM.00667-15

Wiederhold, N. P. (2017). Antifungal resistance: current trends and future strategies to combat. Infection and drug resistance, 10, 249 https://doi.org/10.2147/IDR.S124918

Willger, S. D., Puttikamonkul, S., Kim, K. H., Burritt, J. B., Grahl, N., Metzler, L. J., ... \& Cramer Jr, R. A. (2008). A sterol-regulatory element binding protein is required for cell polarity, hypoxia adaptation, azole drug resistance, and virulence in Aspergillus fumigatus. PLoS Pathog, 4(11), e1000200 https://doi.org/10.1371/journal.ppat.1000200

Xiao, L., Madison, V., Chau, A. S., Loebenberg, D., Palermo, R. E., \& McNicholas, P. M. (2004). Three-dimensional models of wild-type and mutated forms of cytochrome P450 14 $\alpha$-sterol demethylases from Aspergillus fumigatus and Candida albicans provide insights into posaconazole binding. Antimicrobial
Agents
Chemotherapy, $48(2)$
568-574.

http://dx.doi.org/10.1128/AAC.48.2.568-574.2004

Zakaria, A., Osman, M., Dabboussi, F., Rafei, R., Mallat, H., Papon, N., ... \&

Hamze, M. (2020). Recent trends in the epidemiology, diagnosis, treatment, and mechanisms of resistance in clinical Aspergillus species: a general review with a special focus on the Middle Eastern and North African region. Journal of infection and public health, 13(1), 1-10.https://doi.org/10.1016/j.jiph.2019.08.007

Zhang, J., Debets, A. J., Verweij, P. E., Melchers, W. J., Zwaan, B. J., \& Schoustra, S. E. (2015). Asexual sporulation facilitates adaptation: the emergence of azole resistance in Aspergillus fumigatus. Evolution, 69(10), 2573-2586 http://dx.doi.org/10.1111/evo.12763

Zoll, J., Snelders, E., Verweij, P. E., \& Melchers, W. J. (2016). Next-generation sequencing in the mycology lab. Current fungal infection reports, 10(2), 37-42. http://dx.doi.org/10.1007/s12281-016-0253-6 